UDC 811.161.1:81'271

DOI: $10.17223 / 24109266 / 9 / 4$

\title{
KENNZEICHEN DER MÜNDLICHKEIT IN DEN SCHRIFTLICHEN AUSHÄNGEN (IM RUSSISCHEN IM VERGLEICH ZUM DEUTSCHEN)
}

\begin{abstract}
N.A. Karpova
Zusammenfassung. Der vorliegende Artikel stellt das Ergebnis der Untersuchung von russischen Texten der administrativen Aushänge im Vergleich zu den deutschen dar. Es ist wieder stärker im Fokus stehenden Bereich der Textsortenlinguistik zuzuordnen, dabei werden auch Aspekte der natürlichen schriftlichen Rede und der Diskursenanalyse berücksichtigt. Der administrative Aushang ist eine relativ neue Erscheinung, die moderne Kommunikationssituation widerspiegelt. Die Spezifik der Analysemethodik lässt sich durch die Notwendigkeit der Beschreibung aller Aspekte des administrativen Aushanges - sowohl linguistischer als auch außerlinguistischer (Besonderheiten der Platzierung der Anzeige, des Stoffes, auf welchem der Text gedruckt oder geschrieben ist, die Wirkung, die der Text dem Leser gegenüber ausübt) zu erklären. Dabei verfolgen wir das Ziel, die Merkmale der gesprochenen Sprache in den schriftlichen Aushängen zu finden und diese zu beschreiben. Die Analyse ist anhand des im Laufe der Untersuchung entwickelten integrativen Analysemodells vollzogen, das sich auf den Prinzipien der Sprechaktentheorie, Textsortentheorie, Diskursanalyse und Beschreibung von Texten der natürlichen schriftlichen Rede stützt. Das Korpus der Untersuchung betragen Texte der über 400 russischen und deutschen administrativen Aushänge. Administrativer Aushang (auch Ordnungstext) wird in dem vorliegenden Artikel als komplexe Art von Textsorte, die zweierlei Funktionen - informative und handlungsreglementierende - ausübt, die anhand der kurzen schriftlichen Mitteilungen vermittelt wird, die in den öffentlichen Plätzen ausgehängt sind. Das sind Großtexte, die aus Subtextsorten mit je verschiedenen dominanten Textfunktionen bestehen: Verbot, Aufforderung, Bitte usw. Jede Art von handlungsreglementierenden Ordnungstexten kann sprachlich unterschiedlich ausgestaltet sein, dabei tauchen bei bestimmten Umständen die Merkmale der gesprochenen Sprache auf. Für die administrativen Aushänge sind folgende Kennzeichen der Mündlichkeit typisch: Spontaneität, Expressivität, Dialogorientierung, die sprachlichen Taktiken der Danksagung und Erinnerung, auch Verwendung von Androhungen, vulgärer und Schimpfwörter, syntaktische Nichtübereinstimmung der Sätze, Gebrauch von Verbalsätzen. Im Russischen und im Deutschen fallen diese Kriterien nicht immer zusammen, die administrativen Aushänge im Russischen zeigen also eine Spezifik aus.

Schlüsselwörter: Textsorte; administrativer Aushang; textsortenspezifische Merkmale; administrativer Diskurs, sprachliche Ausgestaltung; Mündlichkeit; moderne Kommunikation; natürliche schriftliche Rede.
\end{abstract}

\section{Einführung}

Die moderne Kommunikation wird durch eine bedeutende Verbreitung der Kommunikationsbereiche charakterisiert. So entstehen teils neue 
Textsorten: Webinare, Tweets, Kommentare zu den Fotos in Instagram und andere Arten von Mitteilungen je nach dem Kommunikationsmittel, teils neue Arten etablierter Textsorten, die sich verselbstständigen. Die Grenzen zwischen Text und Diskurs, Mündlichkeit und Schriftlichkeit, Text und Bild werden unschärfer. Besonders auffällig ist diese Tendenz in der sachlichen Kommunikation, im Bereich des öffentlichen Verkehrs, dessen Charakter immer spontaner und ungehemmter wird, zu beobachten. Diese Tendenz äußert sich in der Senkung des Förmlichkeitsgrads bei der Bildung sowohl mündlicher als auch schriftlicher Texte des öffentlichen Verkehrs bzw. des administrativen Diskurses und in der Umnormung der ethischen und sprachlichen Regel, wobei umgangssprachliche Elemente und Kolloquialismen in den administrativen Diskurs durchdringen. Der Gegenstand unserer Untersuchung - der administrative Aushang (auch Ordnung, Gebot-und Verbotszeichen, reglementierend-handlungskontrolierende Texte [1: 194]) - gehört gerade zu diesem Spannungsfeld zwischen dynamischen Tendenzen einerseits und dem Fortbestehen traditionellerer Textsorten andererseits.

\section{Untersuchungsobjekt und Textkorpus}

Administrativer Aushang ist eine Erscheinung, die die Besonderheiten der modernen Kommunikationssituation in vollem Maße widerspiegelt und dementsprechend Interesse von gegenwärtigen Sprachforschern immer wieder weckt (sieh Werke von M.G. Besjaewa, L.B. Bojko, T.M. Bolschakowa, J.N. Eremeew, Ch. Fandrych u. M. Thurmair, N.B. Lebedewa, B.Ju. Norman, S.G. Ter-Minasowa A. Wierzbicka und andere). Es ist eine komplexe Textsorte, die einerseits zur großen Klasse der Textsorte von Anzeigen gehört, andererseits, selbst als Großtext, der aus Subtextsorten mit je verschiedener dominanten Textfunktionen besteht. Als erste soll die informative Funktion genannt werden: "grundsätzlich sind alle Anzeigen wissensbezogene, informative Texte, mit denen konstatierend-assertierend wissen bereitgestellt wird, indem etwas öffentlich bekannt gemacht wird" [Ibid.: 239]. Als zweites ist stark ausgedrückte handlungsbeeinflussende Funktion zu erwähnen, die darin besteht, "das Handeln von Personen in einer möglichst genau definierten Domäne zu reglementieren und damit zu kontrollieren" [Ibid.: 196]. In den Werken der Sprachforscher werden unterschiedliche Fachbegriffe benutzt, die die direktive Orientierung und administrative Textfunktion der erforschenden Erscheinung darstellen. Unseres Erachtens, passt der Begriff "administrativer Aushang" am besten für diese Textsorte, denn damit wird nicht nur ihre Hauptfunktion ausgedrückt, sondern auch auf materielle Form ihrer Existenz hingewiesen.

Administrative Aushänge sind kurze schriftliche Mitteilungen, die in öffentlichen Plätzen platziert sind und auf Reglementieren und Kontrollieren der Handlungen von Adressaten gerichtet werden [2: 94]. Zu den öffentli- 
chen Plätzen gehören vor allem Institutionen, die die Regeln für das Handeln des Nutzers dieser Institution bzw. von Dienstleistungen der Institution formulieren: Bibliotheken, Cafés, Universitäten, Banken, Supermärkte usw. Die Regeln haben häufig rechtlich bindenden Charakter: Ihre Grundlage beruht auf der Autorität der Institution, welche die Ordnung erlässt, die dabei geltende Gesetze und Rechtsprechung berücksichtigt [1: 194]. Zum Beispiel:

(1) Уважаемые читатели! Просим Вас бережно относиться $\kappa$ библиотечным книгам! Напоминаем:

- не делайте пометок на страницах;

- не перегибайте книгу в корешке;

- не загибайте уголки странии;

- не вырывайте листы.

При получении книги проверяйте ее физическое состояние. Сообщайте обо всех замеченных дефектах библиотекарю. Помните: ответственность за порчу несет тот, кто пользовался книгой последним. При утере книги ее следует заменить идентичным экземпляром или возместить восстановительную стоимость (реальную рыночную стоимость книги). www.lib.tsu.ru straft.

(2) Betreten verboten. Lebensgefahr. Zuwiderhandlungen werden be-

Die beiden Anzeigen wurden von den Institutionen in einer Mischung juristischer Fachsprache und der Allgemeinsprache verfasst. Durch die Anrede "Liebe Leser" und spezifische Lexik in der ersten Anzeige äußert sich die Bibliothek als textverfassende Institution. Das zweite Beispiel lässt sich durch einen Nominalstil mit unpersönlichen Formulierungen und elliptischen Sätzen charakterisieren. Der Textverfasser bzw. die Institution wird dabei nicht genannt, aber dem Inhalt nach wird es klar, dass es keine Person ist, die aus privaten Interessen handelt, sondern Vertreter einer Institution.

Für die Zwecke der vorliegenden Arbeit wurden etwa 400 deutsche und russische Texte der administrativen Aushänge untersucht, die teils von der Autorin selbst gesammelt wurden, teils aus dem Internet heruntergeladen waren.

In den meisten Fällen handelt der Textproduzent zu Gunsten einer Institution, so wird der administrative Aushang im Rahmen eines institutionellen administrativen Diskurses betrachtet, der in verschiedenen Bereichen menschlicher Lebens repräsentiert wird. Als Bestandteil institutionellen Diskurses funktioniert der administrative Aushang in medizinischen, sachlichen, pädagogischen und anderen Kommunikationsbereichen und widerspiegelt die Merkmale von diesen, was sich formal und inhaltlich zeigt. Dabei lässt sich der administrative Aushang in allen Kommunikationsbereichen als eine einheitliche Textsorte mit den textsortenspezifischen sprachlichen Merkmalen erkennen. 


\section{Methodisches Vorgehen}

Textsorten und ihnen zugrunde liegenden "Textmuster" haben sich in verschiedenen Sprachgemeinschaften historisch entwickelt, um spezifische kommunikative Aufgaben in der sozialen Handlungspraxis zu bewältigen. Textsorten lassen sich als typische Kombinationen von situativen Faktoren, funktionalen und strukturellen Eigenschaften beschreiben [1: 16]. Textsorte kann als musterhaftig charakterisiert werden: in unserem Bewusstsein erscheinen die Textsorten als Muster der sprachlichen Handlung in einer konkreten kommunikativen Situation [sieh 3]. Zugrunde unserer Untersuchung haben wir das Modell der Beschreibung der Textsorten von T.V. Schmeljowa [4] mit Hinblick auf die spezifischen Merkmale von dem untersuchten Material aufgenommen. Da die Sprache aber ein lebendiges und dynamisches System ist, lassen sich nicht alle Textsorten mit Hilfe eines und desselben Kriterienbündels beschreiben.

Um den administrativen Aushang zu charakterisieren, wichtig ist auch seine materielle, mediale Form und Stelle, wo es ausgehängt wird - also die paraverbalen Kriterien, die mit Textsortenanalyse nichts zu tun haben. Deswegen haben wir uns an ein anderes Modell der Textbeschreibung gewendet u.z. mit dessen Hilfe die Texte der natürlichen schriftlichen Rede sich untersuchen lassen. Den Begriff "natürliche schriftliche Rede" («естественная письменная речь») hat in die Linguistik N.B. Lebedewa eingeführt, die darunter eine Art von Rede versteht, die die Merkmale sowohl schriftlicher als auch mündlicher Rede enthält [5: 173-180]. So wurde im Laufe der Untersuchung ein integratives Analysemodell entwickelt, das sich auf den Prinzipien der Sprechaktentheorie, Textsortentheorie, Diskursanalyse und Beschreibung von Texten der natürlichen schriftlichen Rede stützt. Die Vereinbarung aller dieser Forschungsrichtungen ermöglicht den administrativen Aushang umfassend auf empirischer Basis und in funktionaler Sicht zu beschreiben, sein kommunikatives Potential und verborgene kommunikative Sinne feststellend [2: 10].

Das Analysemodell enthält also linguistische und extralinguistische textsortenkonstitutive Faktoren, dank denen wir eine schriftliche Mitteilung als administrativen Aushang wahrnehmen. $\mathrm{Zu}$ den linguistischen Faktoren der Beschreibung von einem Text des administrativen Aushanges gehören:

1) Textfunktion (reglementierend-direktive);

2) Charakteristika von Textverfasser und Leserschaft bzw. Autor und Rezipient, Adressant und Adressat (es gibt keinen konkreten Adressaten, es liegt die Mehrfachadressiertheit vor. Leserschaft ein größerer Kreis von mehr oder weniger spezifierbaren Personen [1:241]; der Leser des administrativen Aushanges ist meistenteils gleichzeitig die tätige Person; der kommunikative Status des Textverfassers ist meistenteils höher als der des Adressaten, das dem Autor leistet die Handlungen des Adressaten zu reglementieren; es besteht keine Kopräsenz von Autor und Rezipienten, sondern das 
Kommunikat wird für eine räumlich, bzw. zeitlich getrennte, differente Rezeptionssituation verfasst);

3) Textstruktur (Anrede, präskriptive / restriktive / permissive sprachliche Handlung, Unterschrift, Danksagung);

4) spezifische sprachliche Merkmale (Direktheit: wortwörtlicher Ausdruck von der sprachlichen Handlung; sachlich-formaler Charakter; klischierte sprachliche Formel; Expressivitätslosigkeit; elliptische Sätze).

Die extralinguistischen (nichtverbalen / paraverbalen) Faktoren sind:

1) Substratum: materielle Existenzform des Aushanges (ein gelbes Schild in der deutschsprachigen Gegend; ein Din-A-4-Blatt Papier in der russischsprachigen Gegend). Zum Beispiel:
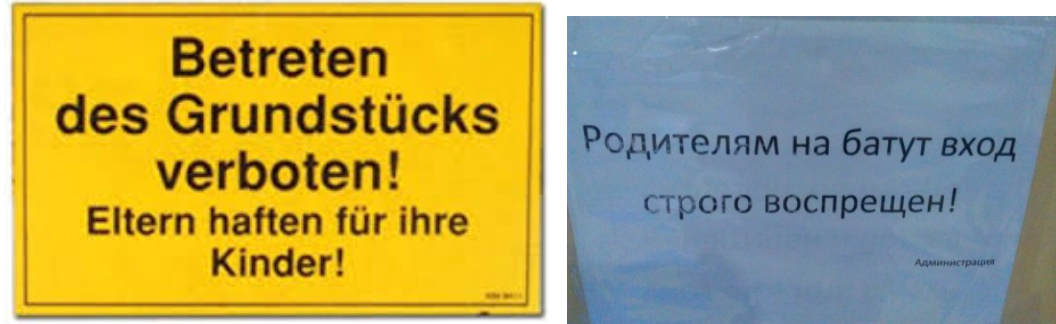

2) Träger des Substrates ("schwarzer Brett" von öffentlichen Einrichtungen, Lehrstuhl, Bauzaun usw.);

3) Grafisch-räumlicher Kriterium des Zeichens (Relation zu den anderen semiotischen Systemen - nonverbale Mittel: farbliche Markierung, Unterstreichung, Besonderheiten des Druckschriftes);

4) Perlokution (bestimmte Handlungen des Adressaten, die als Folge des Lesens von dem Aushang entstehen, also die Wirkung, auf die der Text gerichtet ist). Dieser Aspekt kann im Rahmen dieser Untersuchung nicht beschrieben werden, aber im Kommunikationsprozesses muss berücksichtigt werden.

\section{Untersuchung}

Die administrativen Anzeigen als Genre der natürlichen schriftlichen Rede enthalten die Merkmale sowohl schriftlicher, als auch mündlicher Rede. Das Ziel des vorgelegten Artikels ist es, die Kennzeichen der Mündlichkeit in den administrativen Anzeigen zum Ausdruck zu bringen.

1) Spontaneität, die sich linguistisch und außerlinguistisch äußert. Das erste, was dabei zu erwähnen ist, ist die Auswahl des Stoffes, Substrates für die Anzeige. Da die deutschen Anzeigen meistenteils auf den gelben Blechschildern erscheinen, sind die russischen administrativen Anzeigen normalerweise auf den Din-A-4-Blatt ausgedruckt. Aber es gibt auch Ausnahmen, zum Beispiel: 


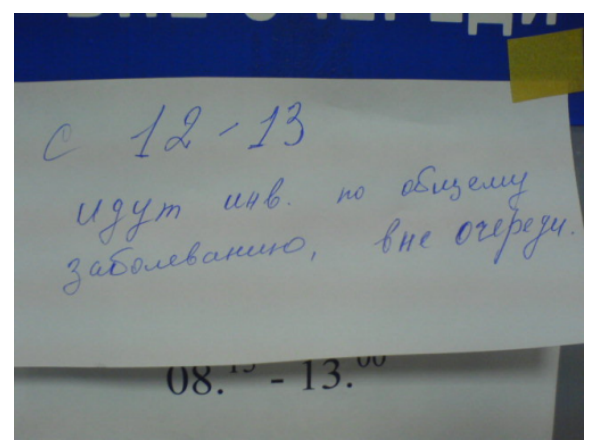

Dieser Aushang lässt sich damit charakterisieren, dass es handschriftlich auf einem kleinen Blatt Papier geschrieben ist. Es enthält keine Unterschrift, wahrscheinlich weil dem Leser der Textverfasser schon bekannt ist dank der Stelle, wo der Aushang belegt ist: die Tür des Raums, wo der Arzt seine Praxis hat. Der Textproduzent möchte den Patienten, die zur Kategorie von Behinderten gehören, damit benachrichtigen, dass sie von 12 bis 13 Recht haben, ohne Reihe zum Arzt zu kommen. Für die anderen ist es eine Vorschrift, den Behinderten ohne Reihe kommen zu lassen. Mag sein, dass sich der Arzt mit dieser Information zuerst mündlich an die in der Reihe wartenden Patienten gewendet hatte, aber nach einigen Fällen des Unverständnisses hat beschlossen, diese Information schriftlich mitzuteilen, oder einfach um Erklärungen zu vermeiden, warum die Behinderten ohne Reihe rein dürfen. Die Art und Weise, wie der Text verfasst wurde (kleines Blatt, keine Anrede und andere Höflichkeitsformeln, Bezeichnung des Adressaten ist abgekürzt) zeugen davon, dass der Autor keine Zeit hatte den Text förmlich zu gestalten, also dieser ist spontan erschienen.

Für die deutschen Aushänge ist die Spontaneität untypisch.

2) Syntaktische Nichtübereinstimmung der Sätze. Dieses Merkmal hängt mit der Spontaneität zusammen und zeugt entweder davon, dass der Textverfasser nicht genug Zeit hatte, den Text richtig vorzubereiten und zu revidieren oder davon, dass der Autor den sachlichen Stil der Rede, den er nachzuvollziehen versucht, nicht beherrscht. Jedenfalls sind die beiden Varianten für Mündlichkeit kennzeichnend. Zum Beispiel:

(3) Уважаемые квартиросъёмщики! Оплачивая коммунальные услуги до 25 числа, Вамей фамилии здесь не будет и Вам не будет стыдно перед соседями.

(4) В связи с ремонтом парикмахерской укладка женщин будет производиться в мужском зале.

Die deutschen Aushänge sind meistenteils sehr sorgfältig formuliert, um gute Verständlichkeit zu garantieren und dadurch Konflikt- und Zweifelfälle zu vermeiden. 
3) Gebrauch von verbalen Sätzen:

(5) Улыбнитесь! Вас снимает скрытая камера:)

(6) Macht mit! Haltet die Toilette (die Dusche) sauber!

Besonderheit dieser Satzart liegt darin, dass sie eine Handlung ausdrücken, die mit einem Subjekt korreliert ist, das Subjekt selbst dabei aber explizit nicht bezeichnet ist [4: 151]. In beiden Belegen ist das Verb im Imperativ gebraucht, das "nicht nur den Prozess, Handlung oder Zustand bezeichnet, sondern Willensäußerung des Autors betreffs Erfüllung/Nichterfüllung der Handlung" von einer anderen Person [6: 13], was auf Adressatenbezug hinweist.

Der Beleg (5) enthält keine direkte Aufforderung, nur die Information, die den Adressaten erinnern soll, dass er videoüberwacht wird und deswegen die in dieser Institution geltenden handlungsreglementierenden Normen zu befolgen hat. Die Allusion zu einer früher sehr populären TV-Sendung wirkt positiv, wobei die Intention des Autors erzielt wird.

Im (6) Beleg ist die Benennung von einem konkretem Adressat vermieden, weil die Aufforderung alle Leser betrifft. Benutzung des Verbes "mitmachen" ist darauf gezielt, den Textrezipienten in eine Gruppe der Menschen mit gleichen Interessen, und zwar der Erhaltung von Sauberkeit, einzuschließen. Als Mitglied dieser Gruppe würde der Adressat kaum gegen den Regeln "seiner" Gruppe verstoßen.

Ein anderes Mittel, das als gruppenkonstituieren bezeichnet werden kann, ist Pronomen wir (vgl. Hoffmann, 1997), und Verwendung des Verbes in Form der 1. Person Plural, zum Beispiel:

(7) Уважсаемые читатели, по вопросам работы зала аудиовизуальных документов просим обращаться к консультантам зала исторических и филологических наук.

In dem gegebenen Fall ist die Bitte mit dem Performativum nрocumb in der Verbindung mit dem Infinitiv des Verbes, das die Handlung bezeichnet, dargestellt, was eher für die gesprochene Sprache typisch ist. Verwendung der persönlichen Form des Verbes weist unvermeidlich auf das Vorhandensein eines konkreten Textautores hin, der über bestimmte personenbezogene und soziale Eigenschaften verfügt, was bei der Rezeption des Textes eine bedeutende Rolle spielt. In der Bibliothek arbeitet ein Team von Fachleuten, die sich bemühen, die Dienstleistungen der Bibliothek zu Gunsten der Leser besser zu machen und die auch Gegenreaktion von diesen erwarten.

4) Adressatenbezug: Anrede, Dialogorientierung. Jeder Kommunikationsakt, sowohl mündlicher, als auch schriftlicher ist auf die Rezeption von einem Adressaten gerichtet [7], je nach dem Adressaten wählt der Textverfasser die Form und sprachliche Ausgestaltung, aber nur in der mündlichen Kommunikation wird der Adressat zur aktiven handelnden Person des Gespräches, an die Fragen gestellt werden, zum Beispiel:

(8) Вошел? Уселся? Рассчитайся!! 
Text des Aushanges besteht hier aus 3 Verbalsätzen, die zwei von denen Fragesätze sind. Die Fragestellung dient hier dazu, die kommunikative Beziehung zu dem Rezipienten aufzubauen und zum Verständnis betreffs der auffordernden Handlung zu appellieren. Die Normen der Sprachetikette schreiben die Verwendung der Form der 2. Person Plural bei der Anrede zu einem unbekannten Menschen im öffentlichen Verkehr vor. In dem Beleg (5) aber wurde eine Du-Form bevorzugt, was einerseits die Formalität der Rede weicht und den kommunikativen Status der Kommunikanten gleichstellt, wobei die Distanz zwischen ihnen reduziert wird und demzufolge der Text auch effektiver wirkt. In diesem Falle hat drückt der verbale Satz die Semantik der Verallgemeinerung: der Textproduzent wendet sich nicht an einem konkreten Adressaten zu, sondern an jedem, der den Text des Aushanges liest.

Noch ein Zeichen für expliziten Adressatenbezug ist die Anrede, wobei sich an den Textrezipienten direkt adressiert wird, zum Beispiel:

(9) Уважаемые гости РК «Шарики»! Самые иенные вещи, документы пусть останутся при Вас! Номерки стоят денег, поэтому беpezume ux!

Auffällig in diesem Beispiel ist auch lexikalische Ausgestaltung und spezifische grammatische Formen: der Text ist vom Standpunkt des Gastes bzw. des Adressaten verfasst, dessen Interessen er scheinbar entspricht. Eigentlich widerspiegelt dieser Aushang eine für Russland ganz typische Kommunikationssituation: die Institutionen, wo es eine Kleiderablage gibt, möchten keine Haftung für den möglichen Verlust von den Wertsachen der Gäste übernehmen und bitten sie deswegen mitnehmen um Konfliktfälle zu vermeiden. Aber in meisten Fällen sieht der Text des Aushanges anders aus:

(10) В гардероб не принимается одежда без вешалок, сумки, пакеты, шарфы, рукавицы. За содержимое карманов ответственности не несем.

Die Textverfasser informieren die Textrezipienten über die in dieser Institution geltenden Normen und lassen eigentlich keine Auswahl: entweder sollen die Wertsachen aus den Taschen ausgenommen werden, oder wird die Kleidung in die Kleiderablage nicht eingenommen. Im Vergleich zu dem (10) Beleg wird im (9) derselbe Inhalt in Form eines Ratschlages ausgestaltet, der dazu noch die Begründung enthält. Dank dieser Taktik wird das Akzeptanzproblem vermieden [11:202].

Die Formulierung mit dem Wort nycmb wird in den schriftlichen Aushängen normalerweise nicht verwendet, weil sie von dem Rezipienten falsch interpretiert werden kann; nicht als eine Aufforderung, sondern als ein Wunsch. In diesem Beispiel aber stimmt die Lexik mit der ausgewählten Vertrauenstaktik überein.

5) Danksagung wird auch im Rahmen der Vertrauenstaktik gebraucht und ist für mündliche Rede charakteristisch. Zum Beispiel: 
(11) Спасибо, что Вы уступили место пожилым людям, пассажирам с детьми и инвалидам.

(12) Bitte keine Werbung danke!

Die Anwendung der Taktik von Danksagen hat zum Zweck, zum einen, die Schärfe der Aufforderung zu mildern. Zum anderen, "Danksagen im Voraus" soll den potenziellen Rezipienten für das Erfüllen des Handels, für welche der Autor sich bei ihm schon bedankt hat, verantwortlich machen. Da das Danksagen schon erledigt ist, soll auch die Handlung rechtfertigt werden. Gestaltung des Aussagens nach den Regeln der Höflichkeit ist scheinbar als Freundlichkeit des Textverfassers zu dem Adressaten als Kommunikationspartner wahrzunehmen, der gleichberechtigte Position im Kommunikationsprozess einnimmt, was auch Akzeptanzproblem vermeiden lässt.

6) Erinnerung ist eine Taktik, die nicht direkt in das Handeln anderer Menschen eingreift, sondern mithilfe der Aktualisierung des beim Adressaten schon vorhandenen Wissens betreffs in dieser Institution geltenden Normen. Zum Beispiel:

(13) При выходе из аудитории не забудьте выключить свет

(14) Selbst Hundebesitzer treten nicht gerne in Hundehaufen. Vergessen Sie also nie, das Geschäft Ihres Vierbeiniges zu beseitigen. Sonst wird es leider teuer. Da sind wir unerbittlich. Stadt Frankfurt am Main.

Sowohl im Deutschen, als auch im Russischen erhalten die Aushänge, die illokutiv als Erinnerung zu lesen sind, durch die Form des Imperativs und der anhand des Verbes im Infinitiv explizit ausgedrückten Handlung den Charakter der Aufforderung. Zum einen wird durch Erinnerung die Wichtigkeit des Ergebnisses der im Aushang bezeichneten Handlung für den Rezipienten selbst veranschaulicht, dem bestimmte Konsequenzen androhen, wenn reglementierende Vorgaben nicht eingehalten werden. Zum anderen verbirgt der Ersatz der direkten Aufforderung durch die Erinnerung die Pflicht des Adressaten die Handlung zu Gunsten des Textverfassers zu erledigen. Der Aufbau bei dem Adressaten einer Illusion, dass seine Meinung von Bedeutung ist, hilft die geplante perlokutive Wirkung zu erzielen.

7) Expressivität wird in den schriftlichen Aushängen mit Hilfe von graphostilistischen Mittel ausgedrückt: Klein-/Großschreibung, Zeichensetzung, Fettdruck usw.

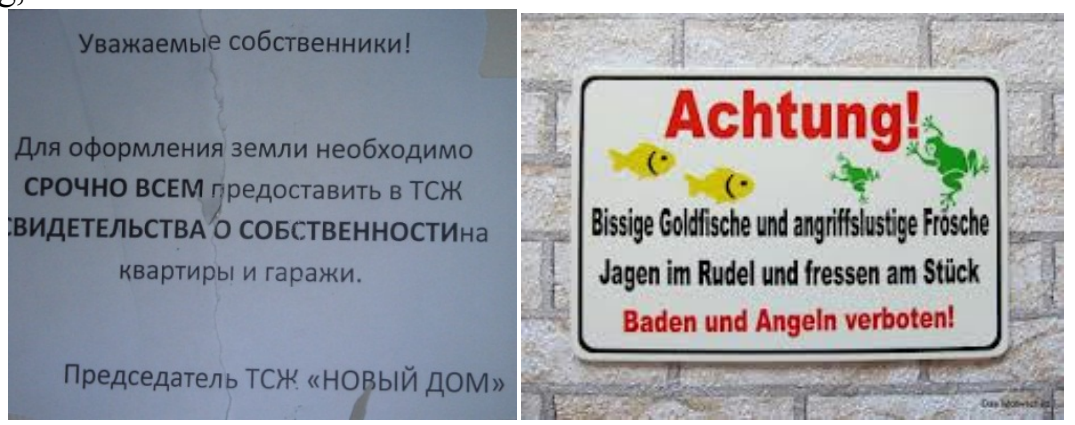


In dem dargestellten Beispiel akzentuiert der Textverfasser einige besonders wichtige Wörter mit Majuskeln. Dabei werden alle Höflichkeitsnormen eingehalten: der Aushang enthält die Anrede zur konkreten Gruppe der Personen, die Unterschrift, und selbst die auffordernde Handlung, die anhand des Wortes срочно verstärkt wird.

Im deutschen Aushang ist die verstärkte Emotionalität anhand der farbigen und schriftlichen Betonung der wichtigsten Information ausgedrückt. Die bunten Bilder auf dem Schild haben zum Zweck die Aufmerksamkeit des Lesers zu ziehen.

8) Androhungen, vulgäre Ausdrücke, Schimpfworte, Jargonismen gehören nicht zur Sprache des öffentlichen Verkehrs und sind normalerweise in den schriftlichen Anzeigen nicht zu treffen. Da aber der administrative Aushang eine schriftliche Textsorte, die durch die Mündlichkeit stark geprägt ist, enthält sie eine andere als normative Lexik, zum Beispiel:
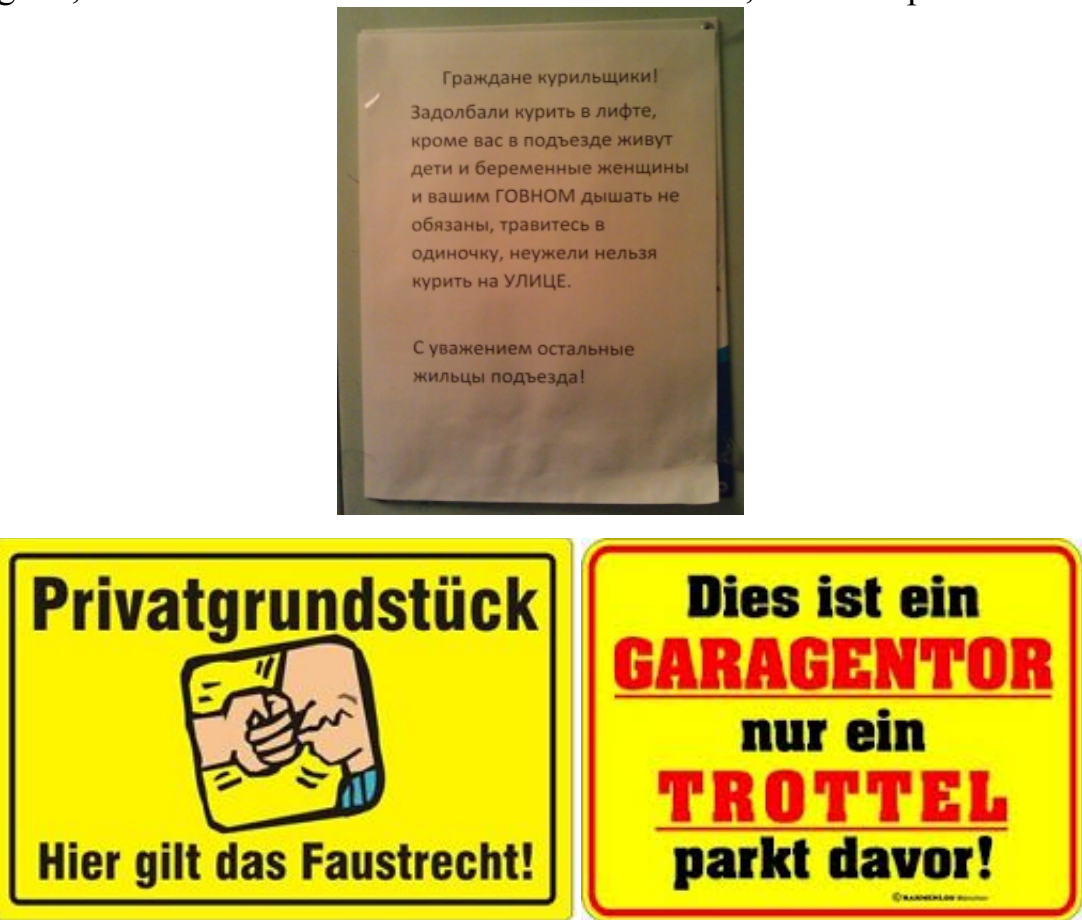

\section{Ergebnisse}

Administrativer Aushang ist eine Textsorte, die zweierlei Funktionen - informative und handlungsreglementierende - ausübt, die durch öffentlich ausgehängte kurze schriftliche Mitteilungen zum Ausdruck gebracht werden. Als Kern der Textsorte administrativer Aushang treten sachlichförmliche Texte, die den Leser zu einer bestimmten Handlung veranlasst und 
der als Bündel verschiedener Merkmale in verschiedenen Typen der Diskursen beschrieben werden kann. Zu den textkonstitutiven Merkmalen gehören: Textfunktion (reglementierend-direktive); Charakteristika von Textverfasser und Leserschaft (der Leser des administrativen Anhanges ist meistenteils gleichzeitig die tätige Person; der kommunikative Status des Textverfassers ist meistenteils höher als der des Adressaten); Textstruktur(Anrede, präskriptive / restriktive / permissive sprachliche Handlung, Unterschrift, Danksagung); spezifische sprachliche Merkmale (Direktheit: wortwörtlicher Ausdruck von der sprachlichen Handlung; sachlich-formaler Charakter; klischierte sprachliche Formel; Expressivitätslosigkeit; elliptische Sätze); Substratum: materielle Existenzform des Aushanges (ein gelber Schild in der deutschsprachigen Gegend; ein Din-A-4-Blatt Papier in der russischsprachigen Gegend); Träger des Substrates ("schwarzes Brett" von öffentlichen Einrichtungen, Lehrstuhl, Bauzaun usw.); grafisch-räumlicher Kriterium des Zeichens (Relation zu den anderen semiotischen Systemen - nonverbale Mittel: farbliche Markierung, Unterstreichung, Besonderheiten der Druckschrift); Perlokution (die extralinguistische Wirkung, auf die der Text gerichtet ist).

So der administrative Aushang lässt sich nicht nur vom Standpunkt der Textsortentheorie, sondern auch im Hinblick zu der materiellen Form und sprachlichen Wirkung erklären, was seine Beschreibung als eines Textes der natürlichen schriftlichen Rede bedingt. In den russischen Aushängen kommen die Merkmale der mündlichen Rede öfter als in den deutschen zum Ausdruck. Für die beiden Sprachen sind solche Kennzeichen der Mündlichkeit wie Expressivität und Dialogorientierung typisch. Diese kommen zum Vorschein durch die besonderen sprachlichen Taktiken der Danksagung und Erinnerung, die der Nähesprachstrategie entsprechen. Es gibt aber auch Texte, in denen eine Konfliktstrategie zum Schwerpunk gelegt wird, die sich in Verwendung von Androhungen, vulgärer und Schimpfwörter äußert. Die Form des Aushanges, seine inhaltliche, formale und materielle Ausgestaltung wird vom Textverfasser in Bezug auf Textrezipienten in einer konkreten Kommunikationssituation ausgewählt. Adressatenbezug wird auch in Gebrauch von für die gesprochene Sprache typischen verbalen Sätzen und in Verwendung von Pronomen wir zur Bezeichnung des Textverfassers geäuBert. Für die russischen administrativen Aushänge ist auch Spontaneität typisch, die den sprachlichen Ausdruck in der syntaktischen Nichtübereinstimmung der Sätze findet, was in der deutschen Sprache kaum der Fall ist. Von der Spontaneität zeugen auch Schriftweise und Auswahl des Stoffes: in diesem Zusammenhang sind handschriftliche Texte auffällig, die nicht auf üblichen A-4 Blätter, sondern auf den nicht dazu geeigneten Stoffen erscheinen. 


\title{
Literature
}

1. Fandrych, Ch., Thurmair, M. (2011). Textsorten im Deutschen. Linguistische Analysen aus sprachdidaktischer Sicht. Tübingen: Stauffenburg Verlag Brigitte Narr GmbH.

2. Karpowa, N.A. (2015). Administrativer Aushang als Textsorte. Textsorten. 1 (11). ss. 93 100.

3. Bakhtin, M.M. (1979). Problem der Textsorten. Ästhetik der verbalen Schöpfung. Moskau. ss. 249-299.

4. Shmeleva, T.V. (1990). Infinitive constructions as functional synonyms of imperative. Functional and typological aspects of imperative analysis. Moscow. pp. 120-137.

5. Lebedewa, N.B. (2007). Genres der natürlichen schriftlichen Rede. Anthologie der Textsorten: alltägliche Kommunikation. Moskau: Labirint. ss. 173-180.

6. Bezyaeva, M.G. (2002). Semantics of the communicative level of the sounding language. Moscow: Moscow State University Press. 752 p.

7. Formanowskaja, N.I. (2007). Adressat in der Sprachkommunikation (SprachkundeKommunikationstheorie). Ausgewählte Artikel. Moskau. ss. 72-83.

Resived 02.05.2017

\section{FEATURES OF THE SPOKEN LANGUAGE IN THE WRITTEN ANNOUNCE- MENTS (IN RUSSIAN COMPARED WITH GERMAN)}

Karpova N.A., Tomsk State University (Russian Federation). E-mail: natakarpova@sibmail.com

\begin{abstract}
The article is a result of research of Russian and German written announcements. As the research material serves an administrative announcement which is regarded as an informative-imperative speech genre mostly represented by short written texts placed in public places and aimed at inducing people to act. AA functions in different communicative spheres: in transport, shop, educational institutions, public catering establishments, on the streets and other public places where there is a need to manage people's behaviour. Depending on the communicative sphere, AA acquires proper lexical content and stylistics. Quite often the text of an announcement is composed in a short time, the conditions of the communicative situation don't allow the author to think long, so the text contains ultimately some features of the oral speech, which we have the aim to reveal and to describe in the present article. AA is a complex phenomenon, a text that combines natural and artificial principles and is aimed at certain extra-linguistic results. Perception of AA depends on material object, substratum (paper, signboard, etc.), type of writing (handwriting, type font, with pictures, highlighting with colour, etc.), location (notice board, transmission tower, wall in the lift, front door, etc.), and language form of expression. So the principles of the analysis include not only linguistic aspects, but also extra-linguistic. For the aims of the present research is used the integrative approach which contains methods of speech genre theory, speech act theory, discourse analysis, natural written language theory. Our research allows to make a conclusion that written announcements, in particular administrative announcements have different grade of officiality and contain some features of the spoken language such as spontaneity, expressivity, dialogic orientation, using of communicative tactics typical for the oral communication: thanking, reminding, threatening, also invectives can be used. At the linguistic level the features of the oral speech show themselves in syntactic incohesion of the sentence, using of verbal sentences. In Russian and in German hang these features not always together.
\end{abstract}

Keywords: speech genre; administrative announcement, specific genre-modelling charakteristics, discourse, spoken language, modern communication, natural written language, language expression

Information about the author:

Karpova Nataliya - Ph.D., Associate Professor, Department of German Language, National Research Tomsk State University (Tomsk, Russian Federation). E-mail: natakarpova@sibmail.com 